\title{
Análisis de los Procesos de Aplicación de las Leyes de Gauss y Ampère por Estudiantes Universitarios de España y Argentina
}

Analysis of the Processes of Application of Gauss's and Ampere's Laws by University Students in Spain and Argentina

\author{
Jenaro Guisasola $^{(1)}$, Julia Salinas ${ }^{(2)}$, José Manuel Almudí $^{(1)}$ y Sandra Velazco ${ }^{(2)}$ \\ (1) Departamento de Física Aplicada I. Universidad del País Vasco. España
}

(2) Departamento de Física. Universidad de Tucumán. Argentina

Recebido em 20 de janeiro, 2003. Aceito em 7 de março, 2003.

\begin{abstract}
El objetivo principal de este trabajo es analizar las formas de razonamiento que presentan los estudiantes universitarios en torno a dos de las leyes del electromagnetismo: la de Gauss (campo eléctrico) y la de Ampère (campo magnético). En ese sentido, hemos supuesto que los déficits observados en la comprensión y utilización de ambas leyes no son consecuencia, únicamente, de la existencia de preconcepciones o de ideas alternativas entre aquéllos sino también del uso habitual de razonamientos basados en la metodología de 'sentido común' que conduciría a razonamientos que determinados autores denominan de 'reducción funcional' y 'fijación funcional'. Para tratar de validar nuestra hipótesis hemos diseñado cinco situaciones problemáticas de tipo abierto con énfasis en las explicaciones. Los resultados obtenidos parecen confirmar que los estudiantes universitarios presentan serias dificultades de aprendizaje en lo que se refiere a las dos leyes analizadas.
\end{abstract}

The main objective of this study is to analyze the reasoning of university students regarding two laws of electromagnetism: Gauss's law (in electric field) and Ampere's law (in magnetic field). It has been supposed that the problems observed in the understanding and application of both laws do not only come from students's misconceptions. Students habitually use reasoning based on 'common sense' methodology that leads to a form of reasoning, known in the literature as 'functional reduction' and 'functional fixation'. To test our hypothesis five open problematic situation, with an emphasis on explanations, were designed. The results obtained seem to confirm that university students have serious difficulties in understanding and applying both of the above mentioned laws.

\section{Introducción}

La asunción de que los estudiantes aprenden mediante una construcción activa de sus propias representaciones es una de las principales premisas de muchas de las investigaciones en el campo de la enseñanza-aprendizaje de las Ciencias [14]. Desde esta perspectiva de aprendizaje constructivista, los estudiantes, frecuentemente, tienden a elaborar su conocimiento dentro de estructuras y marcos teóricos alternativos [5]. Por ello, el conocimiento de las estructuras de comprensión de los estudiantes se ha convertido en uno de los aspectos fundamentales para el diseño de los curricula de ciencias y la elaboración de estrategias de enseñanza.

Sin embargo, a medida que se han ido detectando múltiples concepciones alternativas, se ha considerado que disponer sólo de una amplia descripción no resulta suficiente para colmar el deseo común de interpretaciones más profundas [6,7]. En esta línea se ha intentado inferir cuál podía ser la lógica interna de las argumentaciones de los estudiantes y se ha encontrado que una de las principales dificultades a ser superadas por los estudiantes para una correcta adquisición de los conocimientos científicos no se presenta solamente en la existencia de preconcepciones (ideas previas que posee el estudiante) o ideas alternativas (ideas erróneas que persisten aún después de haber estudiado), sino también en el uso reiterado de razonamientos basados en la metodología de 'sentido común' [8-10]. Esta metodología se caracteriza por la certidumbre, por la ausencia de dudas en su razonamiento y la no consideración de soluciones alternativas, por dar respuestas rápidas y seguras basadas en evidencias de sentido común, así como por tratamientos concretos en los que no preocupa la coherencia de la solución obtenida en un caso con las que se extraen en situaciones bien diferentes.

La existencia de unos modos o patrones de razonamiento podría dar pautas para comprender la resistencia de los estudiantes a cambiar las concepciones alternativas por otras científicas. Los verdaderos obstáculos para este cambio serían ciertos modos de razonamiento bien anclados [7]. 
En definitiva, las perspectivas actuales sobre qué se entiende por aprender ciencias están cambiando. Van quedando atrás aquellas visiones que reducían el aprendizaje de las ciencias exclusivamente a la adquisición de conocimientos teóricos y se incorporan a la enseñanza el conocimiento procedimental y estratégico como aspectos importantes en la práctica de las ciencias [11]. La adquisición de un conocimiento declarativo es un proceso de construcción donde implícita o explícitamente se utiliza el conocimiento procedimental. Los estudiantes pueden memorizar algunos aspectos del contenido declarativo, pero este tipo de aprendizaje no ayudará a mejorar el conocimiento procedimental. La tarea del profesor consiste en enseñar de forma que el estudiante participe en el proceso de construcción, ya que de esta forma mejora la significatividad de los conceptos e incrementa el conocimiento procedimental [12]. Inducción, deducción o inferencia son diferentes formas del conocimiento procedimental. Diferentes procesos mentales complejos (estrategias cognitivas) tales como control de variables (análisis de variables dos a dos) o razonamiento correlativo (comparar porcentajes de acuerdo y desacuerdo en fenómenos) están implicados en el proceso [12].

Vista la necesidad de relacionar íntimamente el aprendizaje de los conceptos teóricos con las habilidades del trabajo científico para que los estudiantes aprendan el cuerpo teórico, será necesario tener en cuenta no sólo sus ideas previas sino también cómo razonan con ellas. Precisamente este trabajo que a continuación presentamos trata de mostrar que estos razonamientos de 'sentido común' se presentan en el dominio del electromagnetismo y, en concreto, en la aplicación de las leyes de Gauss y Ampère dentro de un marco teórico maxwelliano. En nuestro estudio vamos a limitarnos a campos eléctricos y magnéticos estacionarios, es decir, constantes en el tiempo dentro de la teoría clásica del electromagnetismo definida por las leyes de Maxwell. En la teoría de Maxwell, se construye un modelo del mundo real denominado "teoría de campo" que es elaborado por la comunidad científica para explicar la naturaleza. Dentro de este modelo se establecen las leyes de Gauss y Ampère que son de especial importancia para relacionar los aspectos cualitativos de la teoría de campo con aspectos cuantitativos relacionados con las magnitudes físicas definidas (carga, intensidad de campo, flujo ... etc.).

Los profesores universitarios compartimos ampliamente la opinión de que los conceptos utilizados en el área de electromagnetismo son fuente de confusión entre los estudiantes. Esta opinión es confirmada por los escasos trabajos que existen sobre dificultades de aprendizaje en este área para el nivel universitario. En ellos se afirma que los estudiantes tienen dificultades para distinguir entre las magnitudes fuerza y campo $[13,14]$ y que necesitan la acción eléctrica o magnética sobre una carga prueba (en forma de movimiento) para reconocer la existencia de campo $[15,16]$.
Además, la representación gráfica del campo, a través de líneas de campo, presenta confusión entre los estudiantes que tienden a materializarlas como "tubos de fuerza" atribuyéndoles un carácter "real" y no las consideran como modelos gráficos realizados por los científicos para explicar la interacción electromagnética [17-19]. En el trabajo que presentamos trataremos de profundizar en los errores procedimentales de los estudiantes cuando aplican las leyes de Gauss y Ampère $y$, analizaremos las formas de razonamiento que utilizan. Pretendemos contestar a los siguientes interrogantes:

¿Cuáles son la formas de razonamiento que los estudiantes emplean cuando aplican las leyes de Gauss y Ampère?

¿En qué medida los errores cometidos por los estudiantes en el cálculo de las leyes de Gauss y Ampère pueden ser explicados por dificultades procedimentales debidas a la fijación y reducción funcionales como formas de razonamiento espontaneo o de 'sentido común'?

La importancia de las leyes de Gauss y Ampère no viene justificada solamente por la facilidad con que permiten calcular campos, en determinadas situaciones de simetría, frente a las leyes de Coulomb y Biot-Savart, sino que responden a un modelo de campo de la interacción electromagnética. Así, la ley de Gauss es una forma alternativa de expresar la ley de Coulomb sobre las interacciones eléctricas, utilizando el modelo de campo en vez del modelo de acción a distancia. Así mismo, la ley de Ampère es la forma alternativa a la ley de Biot-Savart dentro del marco de campo clásico de las interacciones electromagnéticas definidas por las ecuaciones de Maxwell. Por consiguiente, estas leyes forman parte del conjunto de ecuaciones básicas del electromagnetismo; estas ecuaciones representan un "modelo de campo" que es elaborado por la comunidad científica para explicar las interacciones electromagnéticas en la Naturaleza. Por lo tanto, una clara comprensión de las características indicadas sobre las leyes de Gauss y Ampère es esencial si queremos que los estudiantes las apliquen correctamente y logren un aprendizaje acorde con el marco teórico científico.

La falta de conocimientos sobre las dificultades de aprendizaje de los estudiantes en esta área hace que los profesores nos encontremos con mayores dificultades que en otras áreas a la hora de realizar las secuencias y estrategias de enseñanza. Pensamos que el estudio aquí presentado puede contribuir a clarificar estrategias de enseñanza que permitan a los estudiantes una clara comprensión de las leyes mencionadas. 


\section{La fijación y la reducción funci- onales como formas de razonami- ento espontaneo a tener en cuenta en la enseñanza de la Fisica}

Las concepciones de los estudiantes y sus formas de razonamiento tienen que ser tenidas en cuenta de cara a un aprendizaje comprensivo del conocimiento científico. Desde un punto de vista constructivista es de interés analizar los razonamientos que siguen los estudiantes cuando aprenden ciencias para lograr detectar tanto dificultades epistemológicas como obstáculos en la adquisición de conceptos [7,20]. Los razonamientos espontáneos (o inducidos por la propia enseñanza) pueden ser debidos a una metodología de 'sentido común' $[20,21]$ y a las dificultades detectadas por la psicología cognitiva al estudiar los métodos utilizados por los resolventes de problemas. En este trabajo nos ocuparemos de dos clases de estos razonamientos: la fijación [22] y la reducción funcionales [23].

Una de las características de la metodología de 'sentido común' es la poca importancia que se concede al conocimiento procedimental, ya que lo que importa normalmente es dar una respuesta final como sea. Como ya hemos indicado, estas formas de razonamiento cotidiano tratan de encontrar soluciones rápidas a los problemas y cuestiones mediante una estrategia única que consiste, por lo general, en la aplicación concreta y directa de una 'receta' que va de la teoría a la situación del problema. Esto es, al aprendiz sólo le preocupa la coherencia local entre la teoría y el resultado obtenido; por eso no reflexiona sobre la coherencia global con otros resultados y, sobre todo, con el cuerpo teórico. Por el contrario, los procedimientos científicos suelen ser más ricos y rigurosos. Por ejemplo, en Electromagnetismo, en el análisis de problemas los científicos realizan un análisis preliminar que les lleva a justificar la adopción de un modelo, por ejemplo 'campo', frente a otro modelo, por ejemplo 'acción a distancia'.

Sin embargo, es frecuente encontrar en la enseñanza de la Física modelos de explicación o procedimientos que involuntariamente favorecen el aprendizaje repetitivo de una única estrategia que, muchas veces, no tiene significado para el aprendiz. Si, además, diferentes profesores reiteramos el mismo algoritmo como si estuviéramos p. ej. enseñando memorísticamente a dividir o multiplicar, puede producirse la fijación funcional de este procedimiento. Aunque el procedimiento le permite al alumno llegar a la conclusión final, puede impedir nuevas formas de imaginar otras estrategias $\mathrm{y}$, por tanto, dificulta el pensamiento creativo o productivo [24].

En efecto, es usual en un contexto académico el asociar una colección de enunciados o situaciones problemáticas con aquellas estrategias actualmente aceptadas para obtener una solución satisfactoria. Así, la propuesta de calcular el campo de un conjunto de cargas se asocia frecuentemente con una estrategia de cálculo y con un modelo. Lo que nos interesa señalar es que se obvia, por ser considerado trivial o por cualquier otra razón, un análisis preliminar que conduzca a elegir una clase específica de modelo, ofreciendo implícitamente al alumno la idea de que un problema está siempre indisolublemente unido a un procedimiento de solución único. De esta forma, se produce una fijación a una estrategia en forma de algoritmo tipo que hace que los estudiantes no se planteen la necesidad de justificar el tipo de modelo, estadístico o analítico, que adoptan para analizar un problema. Por ejemplo, los estudios en resolución de problemas en física basados en la psicología del procesamiento de la información indican que "la gran mayoría de los estudiantes resuelven problemas aplicando una estrategia directa que les lleva a una asociación estimulo-respuesta fácilmente recordable por utilizar sólo uno o dos pasos en el proceso" $[25,26]$.

En otras ocasiones pueden presentarse otros modos de razonamientos espontáneos o inducidos que la investigación educativa ha denominado reducción funcional. [23] define este concepto como aquella tendencia a razonar en la que no se toman en consideración todas las variables que influyen en un problema. El caso más común de este tipo de reducción funcional consiste en reducir el número de variables en un problema de múltiples variables, que hace que los estudiantes 'olviden' alguna de las variables fundamentales. Por ejemplo, numerosa bibliografía sobre dificultades en circuitos eléctricos sencillos, explica que los estudiantes sólo tienen en cuenta dos variables de todas las que intervienen en el circuito [27,28]. A propósito de este tipo de razonamiento, [29] encuentra que es como si un efecto que puede ser producido por múltiples causas se reduzca a un causalismo simple del tipo 'un efecto es producido sólo por una causa'.

Otra modalidad de reducción funcional se presenta cuando los estudiantes identifican (es decir, consideran iguales) ciertas variables o conceptos que, si bien están relacionados epistemológicamente, son diferentes.Por ejemplo, cuando los estudiantes identifican dos conceptos diferentes pero epistemológicamente relacionados como velocidad y aceleración en Mecánica [30].

También se considera como reducción funcional el denominado por [31] razonamiento lineal causal, que normalmente se presenta en situaciones de varias variables que requieren argumentos complejos y donde el resolvente va disminuyendo la complejidad del problema a base de construir implicaciones simples del tipo una causa un efecto en forma de cadena lineal, sin ramificaciones en la argumentación, que avanza secuencialmente hasta llegar a la solución final $[6,7]$. 


\section{Diseño experimental}

De acuerdo con las consideraciones anteriores hemos supuesto, a título de hipótesis, que los estudiantes al término de una instrucción tradicional de los temas de electromagnetismo realizarán aplicaciones mecánicas de las leyes de Gauss y de Ampère basadas en un aprendizaje memorístico de las ecuaciones. Así mismo, esperamos que la no utilización de procedimientos científicos (planteamientos cualitativos de las situaciones a analizar, emisión de hipótesis, contrastación experimental de resultados, análisis de los mismos,...etc.) lleve a los estudiantes a utilizar modos de razonamiento como la reducción y la fijación funcionales.

Para tratar de identificar las formas de razonamiento que los estudiantes utilizan al emplear las leyes de Gauss y Ampère hemos diseñado cinco situaciones problemáticas que son frecuentes en los libros de texto utilizados para la enseñanza de la Física General en primeros curso de Universidad. El estudio está orientado hacia estudiantes de dichos cursos. De acuerdo con [32], en primer lugar, hemos utilizado una combinación de diseños cuantitativos (cuestionario) y cualitativos (entrevista) ya que la detección de los razonamientos de los estudiantes incluyen múltiples aspectos que es preciso detectar con una variedad de métodos. En segundo lugar, utilizando de forma coordinada y con el mismo objetivo los dos métodos pueden complementarse y ofrecernos aspectos que sería difícil obtener utilizando sólo uno de ellos. Y en tercer lugar, para validar nuestras hipótesis es necesario utilizar diferentes técnicas que puedan ofrecer resultados convergentes.

109 estudiantes de primer curso de Ingeniería Técnica de la Universidad del País Vasco (España) realizaron el cuestionario. Las preguntas del cuestionario (ver Anexo I) hacen énfasis en las explicaciones de los estudiantes. Las dos primeras cuestiones tratan sobre la ley de Gauss y las dos siguientes sobre la ley de Ampère. Para cada una de las leyes se ha diseñado más de una pregunta. Analizar las respuestas de los estudiantes para una misma ley desde situaciones distintas nos puede facilitar ver cómo razonan [33]. El cuestionario ha sido validado por los autores de este trabajo y tres profesores expertos para analizar los contenidos y sus objetivos. Así mismo, se realizó una primera prueba piloto para analizar la coherencia entre la redacción de las cuestiones y las respuestas de los estudiantes [34]. El cuestionario que se presenta es la última versión que fue aplicada a la muestra citada anteriormente. Debido a su longitud se realizaron dos partes del cuestionario que fueron contestados por dos clases de 44 y 65 alumnos de primero de Ingeniería.

El análisis del cuestionario fue realizado de acuerdo con la revisión bibliografica realizada sobre concepciones de los estudiantes en electromagnetismo y nuestra experiencia como profesores. En primer lugar, uno de los autores de este trabajo realizó un análisis del 10\% de los cuestionarios y estableció algunas formas de razonamiento. Después se discutió con los demás miembros del grupo las formas de razonamiento establecidas y corrigieron la misma muestra por separado, llegándose a un consenso en las categorías de razonamiento del $85 \%$. Después se corrigió la muestra total produciéndose un alto grado de consenso en las categorías de razonamiento. En los casos en que no había coincidencia se llego a un acuerdo por consenso.

Se entrevistó a 12 estudiantes de $2^{\circ}$ curso de Físicas de la Universidad de Tucumán (Argentina) utilizando tres situaciones problemáticas empleadas en el cuestionario (item1, ítem 3 e ítem 4a). Las entrevistas tratan de ver en profundidad cómo los estudiantes aplican las leyes de Gauss y Ampère en estas situaciones. Así mismo, trata de ver si existe convergencia entre estas explicaciones y las dadas por los estudiantes en el cuestionario. Las entrevistas duraron aproximadamente 30 minutos y fueron gravadas en audiotape para su posterior análisis. Las entrevistas se diseñaron con el propósito de que, en primer lugar, los estudiantes propusieran la estrategia a utilizar en la ley de Gauss o de Ampère y la justificaran. En segundo lugar, aplicaban la ley y, en tercer lugar, se les estimulaba a realizar un análisis crítico del resultado y, en su caso, a volver a realizar la cuestión [35]. En definitiva, se ha intentado que los resultados de esta aproximación cualitativa al razonamiento de los estudiantes sean lo más fiable posibles [36].

La muestra está compuesta por 109 estudiantes de primer curso de Ingeniería Técnica de la Universidad del País Vasco (España) y 12 estudiantes de $2^{\circ}$ curso de Físicas de la Universidad de Tucumán (Argentina). Ambos grupos han visto en electromagnetismo el mismo currículo de Física, utilizando libros de texto similares. Sin embargo, los estudiantes argentinos se pueden considerar de mayor nivel ya que han superado el temario de primer curso, mientras que los españoles lo han trabajado para exámenes parciales pero todavía no ha realizado el examen final. Ambos grupos han recibido clases magistrales expositivas dentro del sistema habitual. Así pues, podemos decir que ambos grupos son homogéneos desde el punto de vista del programa y enseñanza recibida. Debido a la disponibilidad de la muestra hemos decidido realizar el cuestionario con los estudiantes españoles y las entrevistas con los argentinos.

\section{Análisis de los resultados}

Para facilitar la presentación de los resultados obtenidos vamos a mostrarlos en diferentes secciones e incluiremos también resultados cualitativos de las entrevistas.

¿Cómo utilizan los estudiantes los operadores de flujo y circulación de campo?

En la primera cuestión se propone una situación en la que el flujo del campo eléctrico a través de una superficie 
cerrada es cero y los estudiantes deben razonar si ello implica que el campo en cada punto de la superficie sea cero. Para una superficie cerrada cualquiera, el flujo eléctrico neto saliente es el producto del promedio de la componente normal del campo según la dirección hacia afuera y el área de la superficie. Por tanto, un razonamiento correcto de la cuestión planteada nos lleva a indicar que la condición $\Phi=0$ no implica necesariamente que el campo sea nulo en cada punto de la superficie, sino sólo que el promedio de las componentes normales a la superficie lo es. Sin embargo, si sólo se sabe que la carga neta encerrada por la superficie es nula, podría ocurrir que existieran cuerpos cargados dentro de la superficie, tal que la suma algebraica de sus cargas eléctricas fuese nula, o que existieran cuerpos cargados fuera de la superficie. En ambos casos el flujo sería cero, pero dichos cuerpos cargados producirán un campo eléctrico no nulo en todo el espacio y, en particular, en cada punto de la superficie gaussiana.

Las respuestas de los estudiantes han sido categorizadas de acuerdo con la tabla 1 que se muestra a continuación. Los resultados que aparecen en esta tabla (también en las sucesivas, hasta que no se diga lo contrario) se darán en porcentaje.

\begin{tabular}{|l|l|}
\hline Categoría de respuesta & Porcentaje de respuestas $(\mathrm{N}=44)$ \\
\hline A: $\Phi=0$ no implica $\mathrm{E}=0$ & 19 \\
\hline B: $\Phi=0$ implica que $\mathrm{E}$ y dS son perpendiculares & 11 \\
\hline C: $\Phi=0$ implica $\mathrm{E}=0$ & 45 \\
\hline Incodificables & 18 \\
\hline No contestan & 7 \\
\hline
\end{tabular}

Tabla 1. Porcentajes de respuesta de los estudiantes en la cuestión 1

En la categoría A hemos agrupado aquellas respuestas que indican que la condición $\phi=0$ no implica necesariamente que el campo sea nulo en cada punto de la superficie gaussiana, sino sólo que el promedio de las componentes normales a la superficie lo es.

En la categoría B se recogen respuestas que indican que la condición $\phi=0$ no implica necesariamente que el campo sea nulo. Un ejemplo estándar de esta categoría de respuestas sería el siguiente:

"El flujo se define como $\phi=\oint \vec{E} \bullet d \vec{S}$ por tanto si los vectores $E$ y $d S$ son perpendiculares el flujo puede ser cero sin que el campo E lo sea"

Se razona de forma puramente algorítmica en base a la fórmula del flujo. Este tipo de razonamiento no permite saber si los conceptos físicos que intervienen son comprendidos correctamente por los estudiantes. Más bien, parece que para los estudiantes el flujo no tiene una entidad propia sino que se utiliza para realizar cálculos. Un razonamiento estandar utilizado por los estudiantes en la categoría C sería el siguiente:

" Si el flujo es cero se cumple que $0=\oint \vec{E} \bullet d \vec{S}$, por tanto el campo en la superficie gaussiana es cero"

Aquí parece que los estudiantes no distinguen significativamente el flujo del campo y establecen una proporcionalidad entre ambos.

En las entrevistas, la mayoría de los estudiantes razonan de acuerdo con la categoría $\mathrm{C}$ y aparecen dos variantes de razonamiento en esta categoría. En una de ellas, los estudiantes no analizan el campo de validez de la ley de Gauss y la utilizan de forma acrítica, tal y se puede ver en una parte de la siguiente entrevista:

Entrevistadora: ¿Por qué dices que el campo en la superficie gaussiana es cero?

Estudiante: Si miramos la ley de Gauss aparece claro. Si el flujo es cero se cumple que esta ecuación: $0=$ $\oint \vec{E} \bullet d \vec{S}=E \oint d S \Rightarrow E=0$, por tanto el campo en la superficie gaussiana es cero

En el razonamiento seguido, los estudiantes no establecen la necesidad de que el campo $\mathbf{E}$ sea constante en módulo a lo largo de la superficie gaussiana y que el ángulo que forma con cada elemento diferencial de área también lo sea, de manera que se pueda sacar fuera de la integral. Esta forma de razonar parece responder a una estrategia ampliamente utilizada en clase pero que tiene un campo de validez muy concreto. Sin embargo los estudiantes la aplican de forma general, produciéndose una fijación funcional a una estrategia.

La segunda variante de razonamiento en la categoría $\mathrm{C}$ (más numerosa que la anterior) agrupa aquellas respuestas que explican que como el flujo es nulo, la carga encerrada será cero y por lo tanto el campo será cero, lo que es coherente con la idea de que sólo las cargas interiores a la superficie gaussiana producen campo eléctrico en los puntos de ella. En este caso parece que los estudiantes simplifican una situación que requiere argumentos complejos en base a construir implicaciones simples del tipo "una causa produce un efecto" en forma de cadena lineal utilizando como vínculo la ley de Gauss (reducción funcional). Veamos un ejemplo de respuesta en una de las entrevistas:

Entrevistadora: ¿Por qué dices que el campo en la su- 
perficie gaussiana es cero?

Estudiante: Si el flujo es cero esto significa que no hay carga ; no?. Pues en la ley de Gauss el flujo es proporcional a la carga y si la carga es cero esto indica que no hay campo. Es decir, si usamos la ley de Gauss, en este caso al ser el flujo cero la carga es cero y no hay campo

En la cuestión $4 \mathrm{~b}$ se plantea lo mismo que en la cuestión 1 pero para el caso del campo magnético y el operador circulación de campo magnético a lo largo de una línea cerrada. En este caso el estudiante debe utilizar procedimientos de tipo metacognitivo para justificar su acuerdo o desacuerdo con la explicación presentada en la cuestión sobre si una circulación de campo magnético a través de una línea cerrada implica la no existencia de campo en los puntos de esa línea. Aquí también una correcta interpretación de la ley de Ampère en el problema planteado lleva a rechazar la argumentación ofrecida. Las respuestas de los estudiantes se presentan en la tabla 2 .

\begin{tabular}{|c|c|}
\hline Categoría de respuesta & $\begin{array}{c}\text { Porcentaje de } \\
\text { respuestas }(\mathrm{N}=65)\end{array}$ \\
\hline $\mathbf{A :} \Phi=0$ no implica $\mathrm{B}=0$ & 18 \\
\hline $\mathbf{C}: \Phi=0$ implica $\mathrm{B}=0$ & 57 \\
\hline Incodificables & 13 \\
\hline No contestan & 12 \\
\hline
\end{tabular}

Tabla 2. Porcentajes de respuesta de los estudiantes en la cuestión $4 \mathrm{~b}$

Respecto a los resultados de esta cuestión es de destacar la convergencia de los razonamientos utilizados con respecto a la cuestión 1. Asi, en la categoría C nos encontramos con explicaciones como la siguiente:

"Si aplicamos la ley de Ampère: $0=$ $\oint \vec{B} \bullet d \vec{l}=B \oint d l \Rightarrow B=0$, yo creo que el estudiante tiene razon"

¿Cómo utilizan los estudiantes las fuentes del campo y su forma cuando aplican las leyes de Gauss y Ampère?

En la cuestión 2 se plantea a los estudiantes un problema de identificación de las fuentes que producen el campo que manejan al aplicar la ley de Gauss. El teorema de Gauss establece que el flujo a través de una superficie cerrada depende solamente de la carga encerrada dentro de la superficie. Como es bien conocido, esta proporcionalidad entre flujo resultante y carga encerrada no es válida en general para la relación entre el campo eléctrico resultante sobre la superficie y la carga encerrada, ya que el campo E resultante en un punto de la superficie se debe tanto a las cargas interiores como exteriores a la superficie gaussiana.

En efecto para el ejemplo de la placa infinita aquí utilizado, debe tenerse en cuenta que el campo producido solamente por las cargas interiores a la superficie $S$ no se calcularía aplicando la ley de Gauss, ya que las variaciones en módulo y dirección de $\mathbf{E}$ en cada punto impedirían encontrar una superficie de integración apta para un cálculo sencillo (ver Fig. 1). Es, en definitiva, la presencia de todas las cargas presentes la que determina la existencia de una simetría especial para el campo eléctrico que permite el tratamiento matemático sencillo al que hemos aludido.

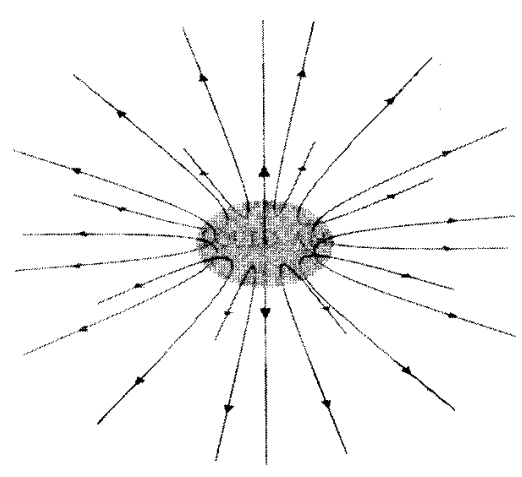

a)

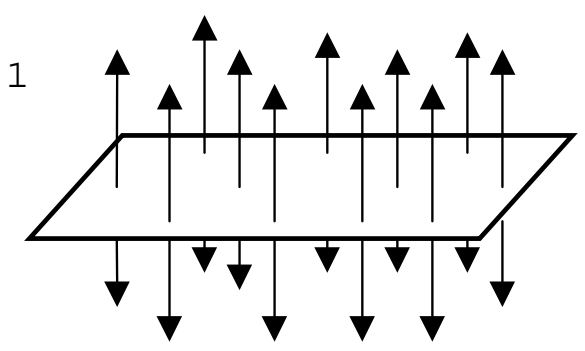

b)

Figura 1. Las líneas de campo producidas a) solamente por la carga interior a la superficie gaussiana, b) todos los elementos de carga de la placa infinita.

Las contestaciones realizadas por los estudiantes se han agrupado por categorías en la tabla 3 .

\begin{tabular}{|l|l|}
\hline Categoría de respuesta & Porcentaje de respuestas $(\mathrm{N}=44)$ \\
\hline A : todas las cargas del espacio generan el campo & 7 \\
\hline B : las cargas interiores generan el campo & 57 \\
\hline C: no justifican la respuesta & 9 \\
\hline Incodificables & 27 \\
\hline
\end{tabular}

Tabla 3. Porcentajes de respuesta de los estudiantes en la cuestión 2. 
En la categoría A hemos agrupado aquellas respuestas que indican que la aplicación de la ley de Gauss exige que es la presencia de todas las cargas presentes la que determina el campo $\mathbf{E}$.

"El campo es el formado tanto por las cargas interiores como exteriores a la superficie gaussiana"

"El campo que se aplica en la ley de Gauss es el formado por las cargas interiores y las exteriores a la gaussiana, aunque en la carga es la interior a la gaussiana"

Sin embargo, en ninguna respuesta se da como justificación el que sólo la presencia de todas las cargas permite la existencia de una simetría especial para el campo eléctrico, que permite el tipo de tratamiento matemático sencillo que conocemos para la ley de Gauss.

En la categoría B la gran mayoria de los razonamientos de los estudiantes son como el siguiente:

"De acuerdo con la ley de Gauss ( $\left.\phi=\frac{Q_{\text {interior }}}{\varepsilon_{0}}\right)$ son las cargas interiores las que crean el campo"

"Si aplicamos la ecuación de la ley de Gauss tenemos que $E=q_{\text {enc }} / \varepsilon_{0} S y$ por tanto es la carga interior la que crea el campo"

En el razonamiento de los estudiantes parece que se vuelve a observar una fijación funcional a una estrategia de resolución basada en la expresión matemática de la ley de Gauss. Parece que a través de la fórmula establecen un vínculo de causalidad entre la carga encerrada por una superficie cualquiera y el campo en los puntos de esa superficie.

Dentro de esta categoría existe otro tipo de razonamiento muy minoritario pero que es preciso constatar. Un ejemplo es el siguiente:

"El campo eléctrico que se calcula por la ley de Gauss es el producido por la carga interior, tal y como indica la fórmula. Pero, como la distribución de carga es uniforme por todo el conductor el campo que se calcula es el de todo el conductor"

Este tipo de explicaciones "ad hoc" estarían asociadas, de acuerdo con la bibliografía, a una "metodología de sentido común" que frecuentemente viene caracterizada por la ausencia de dudas, por respuestas seguras y rápidas basadas en "evidencias de sentido común" y por la falta de consistencia en el análisis de la situación $[21,37,38]$.

En la categoría $\mathrm{C}$ están agrupadas aquellas respuestas que no consideran la ley de Gauss para responder sino que se basan en el principio general de que el campo eléctrico es generado por todas las cargas del plano sin entrar en mayores justificaciones. En nuestra opinión este tipo de respuestas no justifican la afirmación que hacen. Un ejemplo de este tipo de respuesta es:

"El campo que calculamos al aplicar la ley de Gauss es el generado por el plano cargado, ya que la fórmula del campo vale para todo el plano" La cuestión 3 es, en el ámbito del magnetismo, formalmente equivalente a la cuestión 2 (en el área del campo eléctrico) que acabamos de analizar. El tipo de respuestas son convergentes con las de la cuestión 2. Las hemos agrupado en las categorías que aparecen en la tabla 4.

\begin{tabular}{|l|l|}
\hline Categoría de respuesta & Porcentaje de respuestas $(\mathrm{N}=65)$ \\
\hline $\mathbf{A}:$ todas las espiras generan el campo & 9 \\
\hline $\mathbf{B}:$ las espiras interiores generan el campo & 60 \\
\hline $\mathbf{C}:$ no justifican la respuesta & 11 \\
\hline Incodificables & 20 \\
\hline
\end{tabular}

Tabla 4. Porcentajes de respuesta de los estudiantes en la cuestión 3

Las entrevistas realizadas sobre esta cuestión nos permiten analizar con mayor profundidad los procesos utilizados por los estudiantes al aplicar la ley de Ampère en las cuestion 3. Veamos un ejemplo de la categoria B que es común a 9 de los 12 estudiantes entrevistados :

(1) Entrevistadora: ¿Cómo aplicas le ley de Ampère a este caso?

Estudiante: Bueno, es fácil porque el problema me da la curva de integración. Aplico la circulación del campo para esa línea y como ya hemos visto en clase, en los lados verticales y en la parte exterior no hay circulación de B. Por tanto de ahí sacaria el campo $B$ ya que conozco la intensidad que circula por las espiras:

$$
\sum I_{\text {encerrada }}=\oint \vec{B} \bullet d \vec{l} \Rightarrow 10 I=B d
$$

Como hay 10 espiras dentro de la curva de integración he multiplicado la intensidad por 10 y 'd' es la longitud de rectángulo de integración.

(2) Entrevistadora: ¿El campo B que has calculado es el que crea todo el solenoide o sólo las espiras que están dentro de la curva de integración?

Estudiante: La fórmula es para las espiras interiores a la curva de integración, luego éstas son las que crean el сатро $B$

(3) Entrevistadora: Pero, si cogieras una curva de inte- 
gración más grande, por ejemplo cogemos 15 espiras ¿cambiaría el campo magnético obtenido?

Estudiante: Bueno (duda). No lo sé, tendría que calcular (vuelve a dudar)....Creo que no, porque aumentar ía la intensidad de corriente pero también la longitud d de la curva de integración.... Creo que quedaría igual..... Creo que tengo razón ya que en clase hemos visto que la fórmula de $B$ vale para todo el solenoide

(4) Entrevistadora: ¿Entonces el B calculado es el generado por las espiras interiores o las de todo el solenoide?

Estudiante: Vaya pregunta, no me la había planteado....(piensa)...Creo que el $B$ es sólo el creado por la intensidad interior, eso es lo que pone en la fórmula. Pero creo que una vez que se calcula vale para todo el solenoide ya que la intensidad que circula por el cable es la misma.

En la entrevista se confirma que la mayoría de los estudiantes presentan una fijación a la fórmula de la ley de Ampère ('Creo que el B es sólo el creado por la intensidad interior, eso es lo que pone en la fórmula'. Apartado (4)) y que no comprenden el significado físico de la misma. Así mismo, se ha podido constatar que calculan el B (apartado (1)) sin especificar las direcciones del vector de campo y del vector dl de la curva. Parece como si siempre se pudiera dar el caso particular de que B y dl son paralelos y además B constante a lo largo del segmento de curva considerado. Esta forma de razonar ya la hemos constatado en la categoría $\mathrm{C}$ del ítem 1 para la aplicación de la ley de Gauss. Así pues, parece confirmarse una forma de razonamiento que presenta una fijación funcional a un caso particular de resolución de la ecuación, en este caso, de Ampère.

La cuestión 4a se diseñó con la intención de ver los razonamientos de los estudiantes en una situación en la que es necesario tener en cuenta de forma explícita cuál es el campo implicado en la ley de Ampère. De esta forma queríamos constatar que los errores detectados en las cuestiones anteriores (ítems 2 y 3 , P2 de la entrevista) sobre la no consideración de las fuentes del campo que interviene en las leyes de Gauss y Ampère puede llevar a los estudiantes a realizar análisis inadecuados en situaciones problemáticas similares a las académicas. Los resultados de la cuestión 4a se indican en la tabla 5.

\begin{tabular}{|l|l|}
\hline Categoría de respuesta & Porcentaje de respuestas $(\mathrm{N}=65)$ \\
\hline A : Intervienen las dos intensidades de corriente & 9 \\
\hline B : interviene la corriente interior & 66 \\
\hline Incodificables & 13 \\
\hline No contesta & 12 \\
\hline
\end{tabular}

Tabla 5. Porcentajes de respuesta de los estudiantes en la cuestión 4a.

En la categoría A los estudiantes critican la resolución del ejercicio y razonan que es necesario tener en cuenta los campos magnéticos generados por las dos líneas de corriente. Veamos un ejemplo:

“La respuesta no es correcta porque sobre el punto A actúa el campo magnético generado por la corriente de dentro y el campo magnético generado por el otro cable de corriente. Creo que ..., tenemos dos campos magnéticos para poner dentro de la integral. No creo que sea fácil de hacer este ejercicio. La integral creo que es complicada”.

En la categoría B los estudiantes consideran que las únicas fuentes del campo son las que encierran la línea amperiana. Veamos dos ejemplos:

"Estoy de acuerdo ya que

$$
\oint \vec{B} \bullet d \vec{l}=B 2 \pi r=\mu_{0} I \Rightarrow B=\frac{\mu_{0} I^{\prime \prime}}{2 \pi r}
$$

“Estoy de acuerdo. Si aplicamos la ley de Ampère tenemos que el campo B y la intensidad son proporcionales”

Parece que los estudiantes emplean dos formas de razonar que a veces se superponen. Por un lado, la forma de razonar se caracteriza por una fijación funcional a las definiciones operativas de la ley que es utilizada de forma acrítica (ver el ejemplo 1) y se infiere erróneamente que las únicas fuentes del campo son las encerradas por la linea amperiana. Además, de forma más minoritaria, aparecen razonamientos que confunden el campo con el operador circulación de campo (ver ejemplo dos) cayendo en un reduccionismo funcional. Las dos formas de razonamiento también aparecen en la tercera situación planteada en la entrevista.

\section{Conclusiones e implicaciones para la enseñanza}

En el cuerpo teórico de la Física existe una clara distinción entre los operadores de campo que nos permiten obtener información del mismo y el propio campo. Evidentemente, los operadores y el campo están relacionados epistemológicamente pero representan conceptos diferentes. En este sentido, las leyes de Gauss y Ampère establecen relaciones cuantitativas entre los operadores flujo y circulación de campo y las cargas o corrientes encerradas por las superficies o líneas imaginarias tomadas "ad hoc". Sin embargo, los resultados obtenidos parecen indicar que, después de la instrucción, la mayoría de los estudiantes utiliza formas de 
razonamiento erróneas como la reducción y fijaciones funcionales que evidencian una falta de significado de los operadores flujo y circulación de campo y, del propio campo. En el cuadro siguiente hemos intentado resumir las formas de razonamiento que han aparecido en el análisis de resultados.

\begin{tabular}{|c|c|}
\hline Formas de razonamiento no cientifico & Ejemplos de contestaciones estandar \\
\hline $\begin{array}{l}\text { A. Fijación funcional basada en definiciones operativas: } \\
\text { Explicaciones que implícitamente consideran que el campo es } \\
\text { constante en todas las situaciones que se aplican las leyes de } \\
\text { Gauss y/o Ampère }\end{array}$ & $\begin{array}{l}\text { A. } \\
\text { "si } \Phi=0 \text { entonces } \\
0=\oint \vec{E} \bullet d \vec{S}=E \oint d S \Rightarrow E=0 "\end{array}$ \\
\hline $\begin{array}{l}\text { B. Reducciones funcionales basadas en construir implicaciones } \\
\text { simples de fenómenos complejos: } \\
\text { B.1. Cuando el flujo o la circulación es cero se deduce que el } \\
\text { campo es cero en la superficie gaussiana o en la línea amperiana. } \\
\text { B.2. Las únicas fuentes del campo son las encerradas por la su- } \\
\text { perficie gaussiana o la línea amperiana }\end{array}$ & $\begin{array}{l}\text { B.1. "Si el flujo es cero y los vectores E y } \\
\text { dS no son perpendiculares, el campo en la } \\
\text { superficie debe ser cero de acuerdo con la } \\
\text { ley de Gauss: } 0=\oint \vec{E} \bullet d \vec{S} \\
\text { B.2. "En la ley de Ampère se indica que son } \\
\text { las intensidades que atraviesan la línea am- } \\
\text { periana las que crean el campo magnético" }\end{array}$ \\
\hline $\begin{array}{l}\text { C. Elaboración de una explicación 'ad hoc' para cada caso. No } \\
\text { se razona de acuerdo con la búsqueda de generalidad y sistemati- } \\
\text { cidad propias del modo científico, que imponen condiciones más } \\
\text { estrictas y rigurosas. }\end{array}$ & $\begin{array}{l}\text { C. "El campo calculado corresponde sólo } \\
\text { al interior de la superficie gaussiana, pero } \\
\text { como el plano tiene una densidad de carga } \\
\text { uniforme se puede generalizar para el resto } \\
\text { del plano" }\end{array}$ \\
\hline
\end{tabular}

Las deficiencias de razonamiento detectadas sobre aspectos conceptuales básicos en la comprensión del electromagnetismo, como son las leyes de Gauss y Ampère, nos llevan a pensar que será necesario introducir modificaciones en la enseñanza habitual que traten de superar estas dificultades de aprendizaje. Dentro del paradigma constructivista en el que desarrollamos nuestro trabajo [39] es evidente que la enseñanza no debe contener solamente una buena secuenciación y clarificación de los conceptos implicados en el marco teórico, sino incluir aspectos procedimentales y ontológicos a los cuales habrá que referirse. En otras palabras, no se puede esperar que los estudiantes puedan aprender los contenidos conceptuales si no se tienen en cuenta aspectos procedimentales y ontológicos como serían los siguientes:

Los estudiantes tienen que conocer cuáles son los problemas que justifican la introducción de nuevos conceptos. Es necesario que los estudiantes perciban que las leyes de Gauss y Ampère, así como los conceptos implicados, no se introducen de manera arbitraria sino que son "construcciones humanas" que se hacen con carácter tentativo y con el propósito de resolver situaciones problemáticas de interés. En este sentido será importante resaltar el modelo de campo y líneas de campo para explicar la interacción electromagnética y que comprendan también la utilidad de conceptos como flujo y circulación del campo.

Si queremos que los estudiantes vean la construcción de los conocimientos como un proceso dinámico, que puede comportar cambios e incluso replanteamientos globales, consideramos necesario no presentar los conocimientos en su estado final en su forma operativa, sino realizar un proceso que lleve a ver su necesidad y construcción. En este sentido, será necesario que los estudiantes estén familiarizados con el modelo de líneas de campo y con los conceptos que pueden dar información sobre él, como el flujo y la circulación de campo, antes de presentar las leyes de Gauss y Ampère en forma operativa. Con esta manera de abordar el tema se pretende, además, distinguir claramente el concepto de campo del de operador asociado a él (flujo para el campo eléctrico y circulación para el magnético).

Además, tendremos que elaborar una serie de actividades de manera que los estudiantes tengan la ocasión de utilizar las estrategias del trabajo científico como son: analizar la situación problemática que se presenta, concebir hipótesis, diseñar y realizar experimentos, modelizarlos cualitativamente (a través del lenguaje matemático y en su caso gráfico), analizar los resultados obtenidos ... y de esta manera poder utilizar razonamientos acordes con los utilizados por la comunidad científica.

El diseño y elaboración de materiales de enseñanza que concreten los puntos anteriores están constituyendo el objetivo de nuestros próximos trabajos.

\section{Referências}

[1] R.A. Duschl,. Restructuring science education,. In Teacher College Press, Columbia University, New York (1990).Tra- 
ducción en castellano: Renovar la Enseñanza de las Ciencias, Narcea (1997).

[2] K. Tobin, D.J. Tippins, \& A.J. Gallard, Research on instructional strategies for teaching science. In Gabel D.L. (eds), Handbook of Research on Science Teaching and Learning. (New York: Mc Millan Pub. Co.) (1994).

[3] N.J. Nersessian, Sci. \& Educ. 4, 203 (1995).

[4] J.I. Pozo, Ens. de las Cienc. 17, 513 (1999).

[5] H. Pfundt \& R. Duit, Bibliography: Students' alternative frameworks and science education, $6^{\text {th }}$ edition. Kiel. Germany. Institute for Science Education at the University of Kiel (1998).

[6] R. Pintó, J. Aliberas y R Gómez, Ens. de las Cienc. 14, 221 (1996).

[7] J. Salinas, L. Cudmani y M. Pesa, Ens. de las Cienc. 14, 209 (1996).

[8] M.Z. Hashweh, Eur. Jour. of Sci. Educ. 8, 229 (1986).

[9] D. Gil y J. Carrascosa, Eur. Jour. of Sci. Educ. 7, 231(1993).

[10] D. Segura, Ens. de las Cienc. 9, 175 (1991).

[11] National Research Council, National standars science education, Washintong D.C.: National Academy Press (1996).

[12] A.E. Lawson, Research of the acquisition of science knowledge epistemological foundations of cognition, In D. L. Gabel (Ed.), Handbook of research on science teaching and learning, New York: Mcmillan. (1994).

[13] C. Furió y J. Guisasola, Ens. de las Cienc. 17, 441(1999).

[14] L. Viennot \& S. Rainson, Int. Jour. of Sci. Educ.14, 475 (1992).

[15] S. Rainson, G. Transtomer y L. Viennot, Am. J. of Phys. 62, 1026 (1994).

[16] L. Viennot, Raisonner en physique. La part du sens commun, De Boeck Université París (1996).

[17] S. Törnkvist, K.A. Petterson and G. Tranströmer, Am. J. of Phys. 61, 335 (1993).

[18] J. Guisasola, J.M. Almudí y M. Ceberio, Students' ideas about the source of magnetic field, Proceedings of the Second International Conference of the European Science Education Research Association (E.S.E.R.A.), 89 (1999).

[19] M.C. Pocovi and F. Finley, Sci. \& Educ. 11, (2002).
[20] C. Furió y M.L. Calatayud, Sci. Educ. 84, 545 (2000).

[21] D. Gil \& J. Carrascosa, Eur. Jour. of Sci. Educ. 5, 70 (1985).

[22] J.R. Anderson, Cognitive Psychology and its implications, W.H. Freeman and company. New York (1990).

[23] L. Viennot, Aster, 14, 127 (1992).

[24] N. Fredericksen, Rev. of Educ. Res. 54, 363 (1984).

[25] M. Ferguson-Hesler y T. De Jong, Cogn. and Instr. 7, 41(1990).

[26] A. Zohar, Jour. of Res. in Sci. Teach. 32, 1039 (1995).

[27] J. Dupin y S. Joshua, Bullet. de l’Uni. des Physic. 683 (1986).

[28] J.L. Closset, Aster, 14, 143 (1992).

[29] B. Andersson, Eur. Jour. of Sci. Educ. 8, 155 (1986).

[30] J. Carrascosa y D. Gil, Ens. de las Cienc. 10, 314 (1992).

[31] S. Rozier, Le rainsonnement linéaire causal en thermodynamique élémentaire, Thèse, Université París 7, LDPES (1989).

[32] T.D. Cook \& Ch.S. Reichardt, Qualitative and quantitative methods in evaluation research. Sage Publications Inc. (1982).

[33] L. Viennot, A multidimensional approach to characterise a conceptual state in students: the role played by questions, In P. Sillos (Ed.). Procedings of the Second Ph. D. Summer School,. Thessaloniki: Aristotle University of Thessaloniki. (1995).

[34] J. Salinas y S. Velazco, Rev. Esp. de la Fís. 13, 38 (1999).

[35] R. White \& R. Gunstone, Probing understanding. The Palmer Press, London (1992).

[36] K.A. Ericsson y H.A. Simon, Protocol analysis : verbal reports as data, Cambridge, the MIT Press (1984).

[37] A.B. Champagne, R.F. Gunstone y L.E. Klopfer, Instructional consequences of students' knowledge about physical phenomena. Effecting changes in cognitive structures among physics students, In L.H.T. West y A.L. Pines (eds.). Cognitive structures and conceptual change. Orlando, FL: Academic Press (1985).

[38] I.A. Halloun y D. Hestens, Am. J. of Phys. 53, 1056 (1985).

[39] D. Gil et al., Ens. de las Cienc. 17, 503 (1999).

\section{ANEXO I: Enunciado de las situaciones problemáticas}

1. Si el flujo de campo eléctrico a través de una superficie cerrada es cero, ¿eso significa que el campo el éctrico en cada 
punto de la superficie es cero?

sí .......... no .......... no sé

Justifica tu respuesta.

2. Considera una placa infinita, con una densidad superficial de carga $\sigma$. ¿El campo el éctrico que determinas a partir de la ley de Gauss:

a) es el campo producido por todos los elementos de carga de la placa?

sí .......... no .......... no sé

b) es el campo producido por la carga que está dentro de la superficie gaussiana?

sí ........... no ... no sé

Justifica su respuesta.

3. Considera un solenoide infinito por el que circula una corriente I. Como sabes, el campo magn ético en el interior del solenoide puede calcularse utilizando la ley de Ampère, empleando una curva de integración como muestra la figura.

\section{curva de integración}

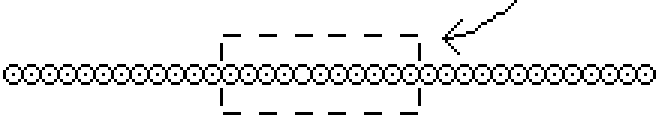

\section{0}

Marca la opción que compartes y justifica tu respuesta:

El campo magnético calculado de ese modo está producido por:

todas las espiras del solenoide

sólo las espiras contenidas en la curva de integración

otra alternativa (explica)

4. Por cada uno de dos hilos "infinitos" pasa una intensidad de corriente de I amperios. Estos hilos son perpendiculares con respecto al plano del papel, estando en uno la corriente dirigida hacia afuera y en el otro hacia adentro. Las trayectorias (1), (2) y (3) son circulares, son recorridas en sentido contrario a las agujas del reloj y encierran, respectivamente, el hilo con la intensidad saliente, el hilo con la intensidad entrante y ambos hilos. La intensidad saliente est á en el centro de (1) y la entrante en el centro de (2).

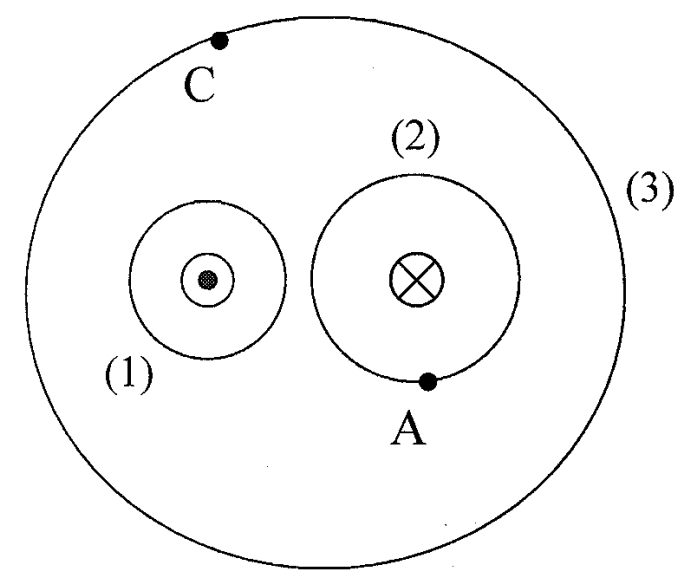

a) Un estudiante utiliza la ley de Ampère y dice que el campo magnético en el punto $A$ es: $B_{A}=\mu_{0} I / 2 \pi r$, siendo $r$ el radio de la trayectoria circular (2). ¿Estás de acuerdo con ese estudiante?.

b) Un estudiante, utilizando la trayectoria (3), aplica la ley de Ampère y llega a la conclusión de que al ser nula la circulación del campo también es nulo el campo B en los puntos de la trayectoria. ¿Estás de acuerdo con ese estudiante?

\section{ANEXO II: Enunciado de los tres problemas para las Entrevistas}

P1. Si el flujo de campo eléctrico a través de una superficie cerrada es cero, ¿eso significa que el campo el éctrico en cada punto de la superficie es cero?

sí... no no sé

Justifica tu respuesta.

P2. Considera un solenoide infinito por el que circula una corriente I. Como sabes, el campo magn ético en el interior del solenoide puede calcularse utilizando la ley de Ampère, empleando una curva de integración como muestra la figura. 


\section{curva de integración \\ 9000000000009000000000000000000000000

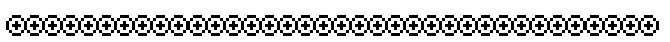

Marca la opción que compartes y justifica tu respuesta:

El campo magnético calculado de ese modo está producido por:

todas las espiras del solenoide

sólo las espiras contenidas en la curva de integración ...........

otra alternativa (explica)

P3. Considera el sistema formado por dos alambres rectos, "infinitamente largos", separados entre sí una distancia " $d$ ", por los que circulan respectivamente las corrientes eléctricas $I_{1}$ e $I_{2}$. Un estudiante afirma que resulta práctico aplicar la ley de Ampère para calcular el campo magnético total en puntos sobre el círculo de radio $R$ marcado en la figura. ¿Estás de acuerdo? Justifica tu respuesta.

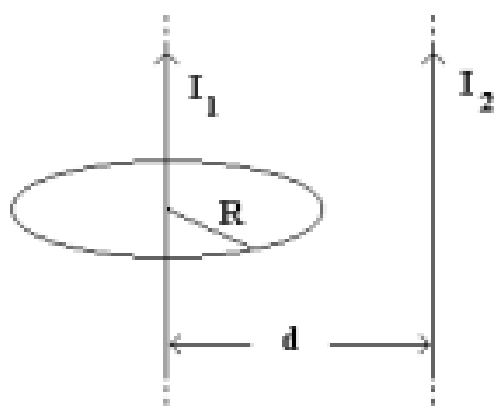

